\title{
HÁBITOS ALIMENTARES E FATORES ASSOCIADOS EM SERVIDORES PÚBLICOS
}

\author{
FOOD HABITS AND ASSOCIATED FACTORS IN PUBLIC SERVANTS
}

\section{João Paulo de Farias ${ }^{a^{*}}$, Waynne Ferreira de Faria ${ }^{b^{*}}$, Antonio Stabelini Neto ${ }^{\mathrm{c}^{*}}$, Aline Miguel de Campos Olímpio ${ }^{\mathrm{d}^{*}}$, Renan Camargo Corrêa ${ }^{\mathrm{e}^{*}}$, Jessica Sartini Sena ${ }^{\mathrm{f}^{*}}$, Rui Gonçalves Marques Elias ${ }^{{ }^{*}}$}

aefjoao@hotmail.com, bfariawf@outlook.com, casneto@uenp.edu.br, daline_miguel12@hotmail.com, erenan_ed91@hotmail.com, fefjoao@hotmail.com, rrgmelias@uenp.edu.br *Universidade Estadual do Norte do Paraná - Jacarezinho (PR), Brasil

\section{RESUMO}

Introdução: A alimentação é um fator fundamental para o funcionamento das atividades físico-biológicas do organismo. Estudos têm evidenciado que múltiplos fatores podem influenciar a ingestáo alimentar do indivíduo. Além disso, a literatura aponta discrepâncias regionais sobre os hábitos alimentares. Dessa forma, identificar os fatores associados aos hábitos alimentares de cada regiáo pode contribuir para direcionar estratégias locais visando a melhora desse quadro. Objetivo: Analisar a prevalência e os fatores associados ao consumo inadequado de frutas, hortaliças, refrigerantes e frituras de servidores públicos da Universidade Estadual do Norte do Paraná (UENP). Métodos: O grupo de estudo foi formado por 147 servidores e professores da UENP do campus Jacarezinho, sendo 73 do sexo feminino e 74 do sexo masculino, com idades entre 18 e 64 anos. Foram avaliados os hábitos alimentares mediante o questionário de frequência alimentar DIPS (Padrão de Consumo Alimentar), nível de atividade física utilizando o questionário IPAQ-8, e nível socioeconômico através do questionário da Associação Brasileira de Empresas de Pesquisa. Resultados: $\mathrm{O}$ aumento da idade mostrou diminuir significativamente o consumo inadequado de frutas, hortaliças, frituras e refrigerantes. A classe socioeconômica baixa-média associou-se ao consumo irregular de frutas e hortaliças $(\mathrm{OR}=2,453$ : $\mathrm{IC}=1,134-0,303$ e $\mathrm{OR}=3,545$ : $\mathrm{IC}=1,370-9,173$, respectivamente). $\mathrm{O}$ consumo inadequado de frutas foi superior para os indivíduos insuficientemente ativos $(\mathrm{OR}=2,683$ : $\mathrm{IC}=1,110-6,489)$. Conclusáo: $\mathrm{A}$ frequência de adultos com hábitos alimentares não saudáveis e a associação destes com a idade, classe socioeconômica e nível de atividade física mostram a necessidade de ações que promovam comportamentos e estilo de vida saudável entre os servidores.

Palavras-chave: Hábitos alimentares; trabalhadores.

\section{ABSTRACT}

Introduction: Food is essential for the functioning of the physical and biological activities of the body. Studies have shown that multiple factors may influence food intake of an individual. Furthermore, the literature indicates regional differences on dietary habits. Thus, identifying factors associated with dietary habits of each region may contribute to direct local strategies to improve this situation. Objective: To analyze the prevalence and factors associated with inadequate intake of fruits, vegetables, soft drinks and frying of public servants of the State University of Northern Parana (UENP). Methods: The study group as formed of 147 servers and teachers from UENP campus of Jacarezinho (PR), 73 females and 74 males aged between 18 and 64 years old. Dietary habits were assessed by the food frequency questionnaire DIPS (Food Consumption Patterns), level of physical activity using the IPAQ 8, and questionnaire and socioeconomic status by the Brazilian Association of Research Companies questionnaire. Results: Increasing age showed significantly decrease the inadequate consumption of fruits, vegetables, frying and sodas. The lowermiddle socioeconomic status was associated with irregular consumption of fruits and vegetables $(\mathrm{OR}=$ 
2,453:CI=1,134-0,303 and $\mathrm{OR}=3,545: \mathrm{CI}=1,370-9,173$, respectively). Inadequate consumption of fruit was higher for insufficiently active individuals $(\mathrm{OR}=2,683: \mathrm{CI}=1,110-6,489)$. Conclusion: The frequency of adults with unhealthy eating habits and their association with age, socioeconomic status and physical activity level shows the need to promote positive behavior and healthy lifestyle among the servers.

Keywords: Food Habits; workers.

\section{Introdução}

A alimentação consiste em um fator fundamental para o funcionamento das atividades físico-biológicas do organismo de um indivíduo ${ }^{1}$. Todavia, a falta de conhecimento sobre as categorias alimentares e necessidades metabólicas diárias, concomitantemente a uma alimentação inadequada, pode se transformar em um fator de risco para o desenvolvimento de doenças crônicas não transmissíveis (DCNT), principalmente quando aliadas a outros fatores de risco ${ }^{2-4}$.

Nessa perspectiva, por serem fontes de micronutrientes, fibras, e apresentarem baixa densidade energética, o consumo adequado de frutas e hortaliças é descrito como um fator protetor contra DCNT ${ }^{2,5-8}$. Além disso, de acordo com a Organização Mundial de Saúde', o consumo insuficiente de frutas e hortaliças está entre os dez principais fatores de risco para DCNT. Dados recentes da Vigilância de fatores de risco e proteçáo para doenças crônicas por inquérito telefônico ${ }^{10}$ (VIGITEL) apontam que na população adulta, apenas $20,2 \%$ dos indivíduos entrevistados consomem frutas e hortaliças de maneira adequada.

Outro fator que pode contribuir para o desenvolvimento de DCNT está relacionado ao consumo exagerado de frituras e refrigerantes, sendo o último responsável por aproximadamente $30 \%$ da ingestão diária de açúcar ${ }^{11}$. Náo obstante, o consumo elevado de frituras e refrigerantes se caracterizam como fatores contribuintes para o desenvolvimento de sobrepeso e obesidade, uma vez que, devido a sua alta concentração energética, esses alimentos poderiam interferir significativamente no equilíbrio entre ingestáo e gasto energético ${ }^{11,12}$. Entretanto, no Brasil são escassas as investigações que buscam analisar os fatores associados ao consumo de frituras e refrigerantes.

Pesquisas têm evidenciado que múltiplos fatores podem influenciar a ingestão alimentar de indivíduos ${ }^{11,13,14}$. Analisando os dados recentes apresentados pelo VIGITEL ${ }^{10}$ sobre o consumo alimentar de indivíduos brasileiros, nota-se grande discrepância entre os dados alimentares de cada região do país. Assim, pesquisas buscando identificar os fatores associados aos hábitos alimentares de cada região podem contribuir para direcionar estratégias e políticas públicas locais, visando à melhora desse quadro, não se restringindo às informações das grandes cidades. Nesse sentido, o objetivo dessa investigação foi analisar a prevalência e os fatores associados ao consumo inadequado de frutas, hortaliças, refrigerantes e frituras de servidores públicos da Universidade Estadual do Norte do Paraná (UENP).

\section{Metodologia}

\section{População e amostra}

Este estudo caracteriza-se como descritivo de corte transversal realizado com adultos da cidade de Jacarezinho (PR). A população foi formada por servidores e professores da UENP do campus Jacarezinho, com a amostra final de 147 servidores, sendo 73 do sexo feminino e 74 do sexo masculino, com idades entre 18 e 64 anos.

O projeto foi aprovado pelo comitê de ética em pesquisa da UENP, processo $n^{\circ}$ 026/2011. Os servidores foram entrevistados e avaliados após receberem explicação dos objetivos do estudo, e após a leitura e assinatura do termo de consentimento livre e esclarecido.

\section{Medidas antropométricas}

A massa corporal (MC) foi mensurada por um único avaliador, utilizando uma balança digital da marca Welmy, com precisão de 100 gramas, e a estatura por meio de um estadiômetro portátil, fixado na parede, graduado de 0 a $200 \mathrm{~cm}$, com escala de precisão 0,1 $\mathrm{cm}$. O IMC foi calculado pela equação da $\mathrm{MC}$ em quilogramas, dividido pela estatura em metros ao quadrado. Os sujeitos foram classificados de acordo com o estado nutricional da seguinte forma: IMC de 18,5 a $24,9 \mathrm{~kg} / \mathrm{m}^{2}$ - peso normal; e acima de $25 \mathrm{~kg} / \mathrm{m}^{2}-$ excesso de peso.

\section{Avaliação comportamental e estilo de vida}

Para os dados referentes à atividade física, foi utilizado o questionário IPAQ-8, versão curta. Foram considerados insuficientes ativos aqueles que não atenderam à recomendação de frequência, duração e intensidade (três ou cinco dias; $150 \mathrm{~min} / \mathrm{sem}$, de moderada a 
vigorosa) de atividades físicas na última semana, e ativos aqueles que atenderam a mesma.

Os hábitos alimentares foram investigados mediante o questionário de frequência alimentar DIPS (Padrão de Consumo Alimentar), recomendado pela OMS ${ }^{15}$ para pesquisas epidemiológicas, traduzido e adaptado para os hábitos alimentares brasileiros ${ }^{16}$. Para este estudo, foram analisados o consumo de frutas, hortaliças, frituras e refrigerantes, na semana anterior à coleta de dados. Adotou-se a seguinte classificação: consumo de frutas e hortaliças < 5 dias/sem - inadequado, e consumo de frituras e refrigerantes $\geq 4$ dias/sem - inadequado.

A avaliação "classe econômica" foi realizada mediante o questionário da Associação Brasileira de Empresas de Pesquisa (ABEP), que considera nove itens relacionados aos bens materiais e à escolaridade, sendo classificados em A, B (classe alta), C, D e E (classe baixa-média) de acordo com a pontuação obtida.

\section{Análises estatísticas}

As variáveis contínuas foram apresentadas em média e intervalo de confiança de 95\% (IC 95\%), e as variáveis qualitativas, em percentuais (\%). Para analisar as variáveis categóricas, aplicou-se o teste de Qui-Quadrado, com correçấo de Yates para variáveis independentes dicotômicas, e o teste de Qui-Quadrado de MantelHaenszel para variáveis categóricas ordinais ou nominais. Como medida de risco, foi calculado o odds ratio, com seus respectivos intervalos de confiança (IC 95\%). Em todas as análises, foi considerado um alfa (p) significativo de 5\%, sendo realizadas no pacote estatístico SPSS, versão 15.0.

\section{Resultados}

As características das variáveis avaliadas dos servidores públicos estão demonstradas na Tabela 1 . A amostra foi composta, em sua maioria, por adultos na faixa etária de $18-29$ anos $(45,6 \%)$, homens $(50,3 \%)$, de classe econômica baixa-média $(71,4 \%)$, com excesso de peso $(51,7 \%)$, e com nível de atividade física insuficiente $(78,9 \%)$. Em relação aos hábitos alimentares, $56,5 \%$, $69,4 \%, 62,6 \%$ e $73,5 \%$ consomem alimentos em frequência adequada para frutas, hortaliças, frituras e refrigerantes, respectivamente.

Tabela 1: Características das variáveis avaliadas dos servidores públicos.

\begin{tabular}{cccc}
\hline Variáveis & N & \% \\
\hline Faixa etária & $18-29$ anos & 67 & 45,6 \\
& $30-39$ anos & 27 & 18,4 \\
& $40-49$ anos & 22 & 21,1 \\
Sexo & $\geq 50$ anos & 31 & 49,7 \\
& Mulheres & 73 & 50,3 \\
Nível socioeconômico & Homens & 74 & 28,6 \\
Estado nutricional & Alta & 42 & 71,4 \\
Nível de atividade física & Baixa-média & 105 & 48,3 \\
& Normal & 71 & 51,7 \\
Consumo de frutas & Excesso de peso & 31 & 21,1 \\
Consumo de hortaliças & Ativo & 116 & 78,9 \\
Consumo de Frituras & Insuficiente ativo & 64 & 43,5 \\
Consumo de Refrigerantes & $<5$ dias/sem & 45 & 30,6 \\
\hline
\end{tabular}

A Tabela 2 apresenta a associação entre o consumo de frutas inadequado e características sociodemográficas, antropométricas, e de atividade física dos servidores. O consumo de frutas $<5$ dias na semana diminui significativamente com avanço da idade $(\mathrm{p}<0,001)$. Os indivíduos de classe socioeconômica baixa-média apresentam maiores chances de consumir frutas em menor frequência, bem como os adultos classificados como insuficiente ativos. 
Tabela 2: Associação entre consumo inadequado de frutas e características sociodemográficas, antropométricas e de atividade física de servidores públicos.

\begin{tabular}{|c|c|c|c|c|}
\hline \multirow{2}{*}{ Variáveis } & & \multicolumn{2}{|c|}{ Frutas $(<5$ dias/sem $)$} & \multirow[t]{2}{*}{ OR (IC 95\%) } \\
\hline & & n (\%) & $\mathbf{P}$ & \\
\hline \multirow[t]{4}{*}{ Faixa etária } & $18-29$ anos & $41(61,2)$ & $0,000 \#$ & \\
\hline & $30-39$ anos & $13(48,1)$ & & \\
\hline & $40-49$ anos & $4(18,2)$ & & \\
\hline & $\geq 50$ anos & $6(19,4)$ & & \\
\hline \multirow[t]{2}{*}{ Sexo } & Feminino & $38(51,4)$ & 0,054 & \multirow{2}{*}{$\begin{array}{c}1,908 \\
(0,646-0,337)\end{array}$} \\
\hline & Masculino & $26(35,6)$ & & \\
\hline \multirow[t]{2}{*}{ Estado nutricional } & Normal & $32(45,1)$ & 0,845 & \multirow{2}{*}{$\begin{array}{c}0,886 \\
(0,462-1,702)\end{array}$} \\
\hline & Excesso de peso & $32(42,1)$ & & \\
\hline \multirow[t]{2}{*}{ Nível socioeconômico } & Alta & $12(28,6)$ & 0,034 & \multirow{2}{*}{$\begin{array}{c}2,453 \\
(1,134-0,303)^{*}\end{array}$} \\
\hline & Baixa-média & $52(49,5)$ & & \\
\hline \multirow[t]{2}{*}{ Nível de atividade física } & Ativo & $8(25,8)$ & 0,042 & \multirow{2}{*}{$\begin{array}{c}2,683 \\
(1,110-6,489)^{*}\end{array}$} \\
\hline & Insuficiente ativo & $56(48,3)$ & & \\
\hline
\end{tabular}

\#=Tendência linear; ${ }^{*}=\mathrm{p}<0,05$.

O consumo de hortaliças e sua associação com as variáveis do estudo estão demonstradas na Tabela 3. Com o aumento da idade, os servidores apresentam maior frequência no consumo de hortaliças ( $\mathrm{p}<0,001)$, e indivíduos de classe baixa-média ingerem hortaliças em menor frequência em relaçáo aos de classe alta $(37,1 \%$ e $14,3 \%)$.

Tabela 3: Associação entre consumo inadequado de hortaliças e características sociodemográficas, antropométricas e de atividade física de servidores públicos.

\begin{tabular}{|c|c|c|c|c|}
\hline \multirow{2}{*}{ Variáveis } & & \multicolumn{2}{|c|}{ Hortaliças ( $<5$ dias/sem) } & \multirow[t]{2}{*}{ OR (IC 95\%) } \\
\hline & & n (\%) & $\mathbf{P}$ & \\
\hline \multirow[t]{4}{*}{ Faixa etária } & $18-29$ anos & $31(46,3)$ & $0,000 \#$ & \\
\hline & $30-39$ anos & $7(25,9)$ & & \\
\hline & $40-49$ anos & $3(13,6)$ & & \\
\hline & $\geq 50$ anos & $4(12,9)$ & & \\
\hline \multirow[t]{2}{*}{ Sexo } & Feminino & $17(23,3)$ & 0,055 & \multirow{2}{*}{$\begin{array}{c}2,005 \\
(0,978-4,110)\end{array}$} \\
\hline & Masculino & $28(37,8)$ & & \\
\hline \multirow[t]{2}{*}{ Estado nutricional } & Normal & $23(32,4)$ & 0,785 & \multirow{2}{*}{$\begin{array}{c}0,850 \\
(0,421-1,716)\end{array}$} \\
\hline & Excesso de peso & $22(28,9)$ & & \\
\hline \multirow[t]{2}{*}{ Nível socioeconômico } & Alta & $6(14,3)$ & 0,012 & \multirow{2}{*}{$\begin{array}{c}3,545 \\
(1,370-9,173)^{*}\end{array}$} \\
\hline & Baixa-média & $39(37,1)$ & & \\
\hline \multirow[t]{2}{*}{ Nível de atividade física } & Ativo & $11(35,5)$ & 0,659 & \multirow{2}{*}{$\begin{array}{c}0,754 \\
(0,326-1,742)\end{array}$} \\
\hline & Insuficiente Ativo & $56(48,3)$ & & \\
\hline
\end{tabular}

\#=Tendência linear; ${ }^{*}=\mathrm{p}<0,05$.

Com o avanço da idade, a frequência de ingestão de frituras diminui entre os servidores $(\mathrm{p}<0,001)$, não sendo encontrada associação significativa nas demais variáveis do estudo (Tabela 4).
A Tabela 5 apresenta a associação entre o consumo inadequado de refrigerantes e características sociodemográficas, antropométricas e de atividade física de trabalhadores públicos. Foi encontrada apenas associaçáo significativa entre a ingestão de refrigerante e faixa etária $(\mathrm{p}=0,036)$. 
Tabela 4: Associação entre consumo inadequado de frituras e características sociodemográficas, antropométricas e de atividade física de servidores públicos.

\begin{tabular}{|c|c|c|c|c|}
\hline \multirow{2}{*}{ Variáveis } & & \multicolumn{2}{|c|}{ Frituras ( $\geq 4$ dias/sem) } & \multirow[t]{2}{*}{ OR (IC 95\%) } \\
\hline & & n (\%) & $\mathbf{P}$ & \\
\hline \multirow[t]{4}{*}{ Faixa etária } & $18-29$ anos & $36(53,7)$ & $0,000 \#$ & \\
\hline & $30-39$ anos & $10(37)$ & & \\
\hline & $40-49$ anos & $3(13,6)$ & & \\
\hline & $\geq 50$ anos & $6(19,4)$ & & \\
\hline \multirow[t]{2}{*}{ Sexo } & Feminino & $33(44,6)$ & 0,070 & \multirow{2}{*}{$\begin{array}{c}1,866 \\
(0,947-3,677)\end{array}$} \\
\hline & Masculino & $22(30,1)$ & & \\
\hline \multirow[t]{2}{*}{ Estado nutricional } & Normal & $32(45,1)$ & 0,093 & \multirow{2}{*}{$\begin{array}{c}0,529 \\
(0,269-1,041)\end{array}$} \\
\hline & Excesso de peso & $23(30,3)$ & & \\
\hline \multirow[t]{2}{*}{ Nível socioeconômico } & Alta & $12(28,6)$ & 0,227 & \multirow{2}{*}{$\begin{array}{c}1,734 \\
(0,799-3,761)\end{array}$} \\
\hline & Baixa-média & $43(41)$ & & \\
\hline \multirow[t]{2}{*}{ Nível de atividade física } & Ativo & $9(29)$ & 0,382 & \multirow{2}{*}{$\begin{array}{c}1,606 \\
(0,680-3797)\end{array}$} \\
\hline & Insuficiente Ativo & $46(39,7)$ & & \\
\hline
\end{tabular}

$\#=$ Tendência linear; ${ }^{*}=\mathrm{p}<0,05$.

Tabela 5: Associação entre consumo de inadequado refrigerantes e características sociodemográficas, antropométricas e de atividade física de servidores públicos.

\begin{tabular}{|c|c|c|c|c|}
\hline \multirow{2}{*}{ Variáveis } & & \multicolumn{2}{|c|}{ Refrigerantes ( $\geq 4$ dias/sem) } & \multirow[t]{2}{*}{ OR (IC 95\%) } \\
\hline & & n (\%) & $\mathbf{P}$ & \\
\hline \multirow[t]{4}{*}{ Faixa etária } & $18-29$ anos & $24(35,8)$ & $0,036 \#$ & \\
\hline & $30-39$ anos & $7(25,9)$ & & \\
\hline & $40-49$ anos & $1(4,5)$ & & \\
\hline & $\geq 50$ anos & $7(22,6)$ & & \\
\hline \multirow[t]{2}{*}{ Sexo } & Feminino & $23(31,1)$ & 0,286 & \multirow{2}{*}{$\begin{array}{c}1,607 \\
(0,765-3,372)\end{array}$} \\
\hline & Masculino & $16(21,9)$ & & \\
\hline \multirow[t]{2}{*}{ Estado nutricional } & Normal & $20(28,2)$ & 0,805 & \multirow{2}{*}{$\begin{array}{c}0,850 \\
(0,408-1,769)\end{array}$} \\
\hline & Excesso de peso & $19(25)$ & & \\
\hline \multirow{2}{*}{$\begin{array}{c}\text { Nível } \\
\text { socioeconômico }\end{array}$} & Alta & $9(21,4)$ & 0,498 & \multirow{2}{*}{$\begin{array}{c}1,467 \\
(0,627-3,431)\end{array}$} \\
\hline & Baixa-média & $30(28,6)$ & & \\
\hline \multirow{2}{*}{$\begin{array}{c}\text { Nível de atividade } \\
\text { Física }\end{array}$} & Ativo & $4(12,9)$ & 0,089 & \multirow{2}{*}{$\begin{array}{c}2,917 \\
(0,949-8,961)\end{array}$} \\
\hline & Insuficiente ativo & $46(39,7)$ & & \\
\hline
\end{tabular}

\#=Tendência linear; ${ }^{*}=\mathrm{p}<0,05$.

\section{Discussão}

A aquisição de hábitos alimentares que consigam suprir as demandas energéticas do nosso corpo, e que se baseiem em alimentos com propriedades preventivas, é hoje considerado um fator essencial para um estilo de vida saudável para pessoas de todas as faixas etárias ${ }^{17}$. Nesta investigação, foi observado que os hábitos alimentares inadequados estiveram associados com idade, classe socioeconômica e nível de atividade física.
Nossos dados revelaram que $43,5 \%$ e $30,6 \%$ dos indivíduos consomem uma quantidade inadequada de frutas e hortaliças, respectivamente. Esses dados são consistentes com os apresentados por Ramalho et al. ${ }^{14}$, que encontraram consumo regular de $20,3 \%$ de frutas e $40,0 \%$ de hortaliças entre universitários. Adicionalmente, outros estudos na literatura que investigaram o consumo de frutas e hortaliças mostraram baixa prevalência de consumo adequado desses alimen$\operatorname{tos}^{8,18-21}$. A recomendaçáo para o consumo de frutas e hortaliças equivale a menos de $400 \mathrm{~g} / \mathrm{dia}$ ou cerca de 
$7 \%$ a $8 \%$ do valor calórico de uma dieta de $2200 \mathrm{kcal} /$ dia. Ademais, o consumo adequado desses alimentos ( $\geq 400 \mathrm{~g} / \mathrm{dia}$ ) é um importante fator protetor contra as DCNT, por serem fontes de micronutrientes, fibras e outros componentes, e também por sua baixa densidade energética ${ }^{8}$.

Com relação ao consumo de frituras e refrigerantes, estudos recentes mostraram que alimentos ricos em açúcares e gorduras estáo inversamente relacionados ao consumo de frutas e vegetais ${ }^{22,23}$. No Brasil, estudos que avaliaram o consumo de frituras e refrigerantes na população brasileira são limitados. Nesta investigação, os resultados mostraram consumo inadequado de frituras e refrigerantes para $62,6 \%$ e $73,5 \%$ dos sujeitos, respectivamente. Resultados semelhantes aos do presente estudo foram apresentados por Zanini et al. ${ }^{13}$, no qual foi apontado o consumo diário de refrigerantes, doces e/ ou frituras para $60 \%$ dos entrevistados, sendo que $10 \%$ relataram consumo diário dos três grupos de alimentos. Esses dados apresentados são preocupantes, tendo em vista que o consumo irregular desses alimentos torna-se um fator de risco para o desenvolvimento de diversas DCNT, como o câncer, diabetes, doenças cardiovasculares, entre outros ${ }^{11,12}$.

Analisando os resultados por faixa etária, observou-se a tendência de menor frequência de consumo de alimentos não saudáveis com o avanço da idade. , Outros estudos também têm verificado tendências nessa direçãa ${ }^{8,14,20}$. A influência da idade sobre a qualidade da dieta pode refletir uma mudança de consciência sobre a saúde com o aumento da idade, interferindo diretamente nas escolhas alimentares pelo grupo de maior idade $^{20}$.

$\mathrm{O}$ excesso de peso não apresentou associação com o consumo frequente dos alimentos investigados neste estudo. Todavia, o estudo de Dinour et al..$^{24}$ demonstrou associação entre os hábitos alimentares e os índices de obesidade, principalmente em mulheres. A falta de significância no presente estudo sugere que, neste caso, possa haver causalidade reversa, dado que, uma vez diagnosticado o excesso de peso corporal, o indivíduo pode ter iniciado medidas de restrição alimentar para o controle desses eventos. Tais características não são passíveis de serem avaliadas em estudos que adotam desenho de corte transversal ${ }^{25}$.

Em relação à classe econômica, nossos resultados demonstraram que a qualidade da dieta é inferior nos indivíduos de classe mais baixa. Resultados semelhantes foram relatados por outros estudos que observaram aumento do consumo de alimentos saudáveis de acordo com a classe socioeconômica ${ }^{14,26}$. O motivo pelo qual a classe econômica mais alta consome dietas saudáveis não foi completamente elucidado, mas pode ser causado pela difusão de conhecimento, atitudes e experiências, além do maior poder aquisitivo para escolha de alimentos ${ }^{27}$.

Estudos internacionais reportaram associaçóes significativas entre prática de atividade física e hábitos alimentares ${ }^{28,29}$. A prática de atividade física foi associada à maior frequência de consumo de frutas, suco de frutas, legumes e verduras, e ao decréscimo do consumo de gorduras totais e saturadas. No presente estudo, esta associação apenas foi verificada para o consumo de frutas.

Como em todo estudo transversal, as associações encontradas não indicam causalidade, e o método autorrelatado apresenta algumas limitaçóes, por exemplo, de indivíduos que podem subestimar seu consumo de alimentos, relatando hábitos "socialmente saudáveis". Contudo, conhecer as características associadas a comportamentos saudáveis é fundamental para identificar grupos com necessidades específicas e orientar políticas públicas, estabelecendo o monitoramento adequado desses comportamentos.

\section{Conclusão}

A alta prevalência de hábitos alimentares não saudáveis, a associaçáo destes com a idade, classe socioeconômica baixa-média, e atividade física insuficiente, mostram a necessidade de açóes que promovam comportamentos saudáveis entre os servidores públicos. Sendo assim, sugerem-se orientaçóes nutricionais, exames de rotina e sessóes de exercícios físicos, visando mudar o estilo de vida desses indivíduos.

\section{Agradecimentos}

Agradecemos a Fundação Araucária, pela concessão da bolsa de iniciação científica, e ao Grupo de Pesquisa em Estilo de Vida, Exercício e Saúde (GPEVES).

\section{Referências}

1. Angelis RC De. Novos conceitos em nutrição. Reflexôes a respeito do elo dieta e saúde. Arq Gastroenterol. 2001;38(4):269-71.

2. Attorp A, Scott JE, Yew AC, Rhodes RE, Barr SI, Naylor P-J. Associations between socioeconomic, parental and home environment factors and fruit and vegetable consumption of children in grades five and six in British Columbia, Canada. BMC Public Health. 2014;fev. 11;14(1):150.

3. Neumann AICP, Martins IS, Marcopito LF, Araujo EAC. Padróes alimentares associados a fatores de risco para doenças cardiovasculares entre residentes de um município brasileiro. Rev Panam Salud Pública. 2007;22(5):329-39. 
4. Fisberg RM, archioni, Maria DL, Colucci ACA. Avaliação do consumo alimentar e da ingestão de nutrientes na prática clínica. Arq Bras Endocrinol Metabol. 2009;53(5):617-24.

5. Santos IS, Duarte EC. Fatores de risco e proteção para doenças crônicas não transmissíveis na populaçáo adulta brasileira. 2009;43(sup.2):5-6.

6. Shigihara M, Obara T, Nagai M, Sugawara Y, Watanabe T, Kakizaki M, et al. Consumption of fruits, vegetables, and seaweeds (sea vegetables) and pancreatic cancer risk: The Ohsaki Cohort Study. Cancer Epidemiol. 2014 fev. 9;38(2):129-36.

7. Cho SW, Kim JH, Lee SM, Lee SM, Choi EJ, Jeong J, et al. Effect of 8-week nutrition counseling to increase phytochemical rich fruit and vegetable consumption in korean breast cancer patients : a randomized controlled trial. Clin Nutr Res. 2014;3:39-47.

8. Jaime PC, Figueiredo ICR, Moura EC, Malta DC. Fatores associados ao consumo de frutas e hortaliças no Brasil, 2006. Rev Saúde Pública. 2009;43(sup.2):57-64.

9. World health organization. The world health report 2002. Reducing risks, promoting healthy life; Geneva: World Health Organization; 2002.

10. Secretaria de vigilância em saúde/secretaria de gestão estratégica e participativa ministério da saúde. VIGITEL Brasil 2011: vigilância de fatores de risco e proteção para doenças crônicas por inquérito telefônico. Brasília: Ministério da Saúde; 2012.

11. Overby NC, Lillegaard ITL, Johansson L, Andersen LF. High intake of added sugar among Norwegian children and adolescents. Public Health Nutr. 2004;7(2):285-93.

12. Miura K, Giskes K, Turrell G. Socio-economic differences in takeaway food consumption among adults. Public Health Nutr. 2012;15(2):218-26.

13. Zanini RDV, Muniz LC, Schneider BC, Tassitano RM, Feitosa WMN, Gonzalez-Chica DA. Consumo diário de refrigerantes, doces e frituras em adolescentes do Nordeste brasileiro. Cien Saude Colet. 2013;18(12):3739-50.

14. Ramalho AA, Dalamaria T, Souza OF. Consumo regular de frutas e hortaliças por estudantes universitários em Rio Branco, Acre, Brasil : prevalência e fatores associados. Cad Saúde Pública. 2012;28(7):1405-13.

15. World Health organization. Global School-based Student Health Survey [Internet]. 2004. Dispnoível em: <http:// www.who. int/school_youth_health/media/en/gshs_chile_ questionnaire2004.pdf> [acesso em 10 out. 2013].

16. Romanzini M, Reichert FF, Lopes A da S, Petroski EL, Farias Junior JC. Prevalência de fatores de risco cardiovascular em adolescentes. Cad Debate. 2008;24(11):2573-81.
17. Viana V. Psicologia, saúde e nutrição: Contributo para o estudo do comportamento alimentar. Análise Psicológica. 2002;4:611-24.

18. Alves HJ, Boog MCF. Representaçôes sobre o consumo de frutas, verduras e legumes entre fruticultores de zona rural. Rev Nutr. 2008;21(6):705-15.

19. Viebig RF, Valero MP, Scazufca M, Menezes PR. Consumo de frutas e hortaliças por idosos de baixa renda na cidade de São Paulo. Rev Saúde Pública. 2009;43(5):806-13.

20. Jaime PC, Monteiro CA. Consumo de frutas e hortaliças na população adulta brasileira, 2003. Cad Saude Publica. 2005;21(s-19):19-24.

21. Toral N, Slater B, Silca MV. Consumo alimentar e excesso de peso de adolescentes de Piracicaba, São Paulo. Rev Nutr. 2007;20(5):449-59.

22. Figueiredo ICR, Jaime PC, Monteiro CA. Fatores associados ao consumo de frutas, legumes e verduras em adultos da cidade de Sáo Paulo Factor. Rev Saúde Pública. 2008;42(5):777-85.

23. Forshee RA, Storey ML. The role of added sugars in the diet quality of children and adolescents. J Am Coll Nutr. 2001;20(1):32-43

24. Dinour LM, Bergen D, Yeh M-C. The food insecurity-obesity paradox: a review of the literature and the role food stamps may play. J Am Diet Assoc. 2007 Nov;107(11):1952-61.

25. Oliveira LPM, Assis AMO, Silva M da Co, Santana MLP, Santos NS, Pinheiro SMC, et al. Fatores associados a excesso de peso e concentração de gordura abdominal em adultos na cidade de Salvador, Bahia, Brasil. Factors associated with overweight and abdominal fat in adults in Salvador, Bahia State, Brazil. Cad Saúde Pública. 2009;25(3):570-82.

26. Campos MTFS, Monteiro JBR, Ornelas APRC. Fatores que afetam o consumo alimentar e a nutriçáo do idoso. Rev Nutr. 2000;13(2):157-65.

27. Popkin BM. Dynamics of the nutrition transition and its implications for the developing world. Forum Nutr. 2003;56:262-4

28. Gillman MW, Pinto BM, Tennstedt S, Glanz K, Marcus B, Friedman RH. Relationships of physical activity with dietary behaviors among adults. Prev Med (Baltim). 2001;32(3):295-301.

29. Dutton GR, Napolitano MA, Whiteley JA, Marcus BH. Is physical activity a gateway behavior for diet? Findings from a physical activity trial. Prev Med (Baltim). 2008 Apr;46(3):216-21. 